


\section{INSTRUCTIONS FOR AUTHORS}

\section{Submission of papers}

Authors are encouraged to submit their original manuscripts online via the website: http://journals.cambridge.org/jom or http://www.editorialmanager.com/jmech/default.asp, where electronic submission and complete instructions for the preparation of manuscripts can be found.

The Journal employs a peer review system in the processing of manuscripts submitted for publication. Each manuscript is sent to reviewers (usually two or more) who are experts in the related fields. Decision as to the publication of the paper is based on the opinions expressed by the reviewers and the judgment of the Editorial board. Reviewers' suggestions for the revision of the manuscript are passed on to the author (s), who is entitled to make use of them or rebut them as he or she sees fit.

If there are any questions with regard to manuscript submission, please contact: jom@pme.nthu.edu.tw

\section{Manuscript preparation}

Papers should be adapted to submission template, at http://journals.cambridge.org/images/fileUpload/documents/Sub mission_Template.docx, and conform to the following instructions:

- Language: The manuscript should be written in good English. It should have been carefully checked for clarity, conciseness, correctness of grammar, and typographical errors.

- Length: A full length paper or review including figures and tables should not normally exceed 16 pages.

- Format: The main divisions are suggested to be arranged as follows: 1. Title page (containing: article, title, author (s), affiliation (s), and corresponding author's address, phone number, fax number and email address); 2. Abstract (of 200 words or less); 3. Keywords (of 4 or less); 4. Main text (containing: introduction, methods of solution, results and discussion, conclusion): 5. Acknowledgements; 6. Appendices; 7. Nomenclature; 8. References. Abstracts are not required for short papers.

- Figures: All photographs, charts and diagrams are to be referred to as "Figures". Captions to figures should be typed consecutively on a separate page (s) at the end of the paper. The preferred format for figure files is .eps or .tiff at a minimum resolution of $1200 \mathrm{dpi}$ for lines, $600 \mathrm{dpi}$ for greyscale and 400 dpi for color. Color art is free of charge for online publication. If figures will be printed in black and white, please ensure that the main information will be visible and do not refer to color in the text.

- Tables: Tables should be typed as part of the text, but in such a way as to avoid confusion with the text. Authors should try to ensure that a single table does not overlay on to the next page. All tables should have headings and be numbered.

- Equations: Mathematical expressions should be consecutively numbered throughout the body of the paper at the right-hand margin in parentheses. Numbering starts anew with each appendix: Appendix A: (A1), (A2), etc., Appendix B: (B1), (B2), etc. Equation numbers mentioned in the text should be enclosed in parentheses, i.e. Eq. (1), Eqs. (1), (2). And use only Word Equation Editor or MathType to produce equations.

- Units: Use of the international system units (SI units) is obligatory. Wherever possible, equations should be written in dimension form.
- References: References should be indicated in square brackets according to the order of appearances in the text, i.e. [1,2-4]. The full list should be collected at the end of the paper in numerical order. Examples of layout of references are given below.

1. Brown, H. E., Amstead, B. H. and Short, E., "Temperature and Velocity Distribution and Transfer of Heat in a Liquid Metal," Journal of Heat Transfer, 79, pp. 279-285 (1957).

2. Zienkiewicz, O. C., The Finite Element Method, 3rd Edition, McGraw-Hill, Maiden Head, England, pp. 45-48 (1977).

3. Zengerle, R., Richter, A. and Sandmaier, H., "A Micro Membrane Pump with Electrostatic Actuation," Proceedings of Micro Electro Mechanical Systems Conference, Germany (1992).

4. Kobayashi, H., "Optimization of Elastic Structure," M. S. Thesis, Department of Aeronautics and Astronautics, Massachusetts Institute of Technology, Massachusetts, U.S.A. (1972).

- Acknowledgements and financial support: Authors are required to add Acknowledgement section and Financial Support section before References if any.

\section{After acceptance}

The corresponding author will be notified by the Editor-inChief of the Journal upon acceptance of the article and invited to supply an electronic version of the accepted manuscript. In the course of the production process, the corresponding author will be asked to transfer the copyright of the article to the Society. This transfer will ensure the widest possible dissemination of information.

\section{Page charges}

There will be no page charges for contributions from outside of Taiwan.

For contributions from Taiwan, a regular page charge of NT\$500 per page will be assessed for articles within 8 published pages (full length papers) or 4 published pages (technical notes). In addition, an excess page fee of NT\$2,000 per each exceeding page will also be charged to the author (s).

\section{PDF offprint}

An author is entitled to a PDF offprint of the published paper free of charge. The PDF offprint will be sent to the corresponding author at the email address supplied on submission. Print offprints may be ordered separately (in multiples of 50).

\section{Author language services}

Cambridge recommends that authors have their manuscripts checked by an English language native speaker before submission; this will ensure that submissions are judged at peer review exclusively on academic merit. We list a number of third-party services specializing in language editing and/or translation, and suggest that authors contact as appropriate. Use of any of these services is voluntary, and at the author's own expense. 